\title{
TU/e EN⿴HONE

\section{Equilibrated charge carrier populations govern steady-state nongeminate recombination in disordered organic solar cells}

\section{Citation for published version (APA):}

Roland, S., Kniepert, J., Love, J. A., Negi, V., Liu, F., Bobbert, P., Melianas, A., Kemerink, M., Hofacker, A., \& Neher, D. (2019). Equilibrated charge carrier populations govern steady-state nongeminate recombination in disordered organic solar cells. The Journal of Physical Chemistry Letters, 10(6), 1374-1381.

https://doi.org/10.1021/acs.jpclett.9b00516

\section{Document license:}

TAVERNE

DOI:

10.1021/acs.jpclett.9b00516

Document status and date:

Published: 21/03/2019

\section{Document Version:}

Publisher's PDF, also known as Version of Record (includes final page, issue and volume numbers)

\section{Please check the document version of this publication:}

- A submitted manuscript is the version of the article upon submission and before peer-review. There can be important differences between the submitted version and the official published version of record. People interested in the research are advised to contact the author for the final version of the publication, or visit the $\mathrm{DOI}$ to the publisher's website.

- The final author version and the galley proof are versions of the publication after peer review.

- The final published version features the final layout of the paper including the volume, issue and page numbers.

Link to publication

\section{General rights}

Copyright and moral rights for the publications made accessible in the public portal are retained by the authors and/or other copyright owners and it is a condition of accessing publications that users recognise and abide by the legal requirements associated with these rights.

- Users may download and print one copy of any publication from the public portal for the purpose of private study or research.

- You may not further distribute the material or use it for any profit-making activity or commercial gain

- You may freely distribute the URL identifying the publication in the public portal.

If the publication is distributed under the terms of Article 25fa of the Dutch Copyright Act, indicated by the "Taverne" license above, please follow below link for the End User Agreement:

www.tue.nl/taverne

Take down policy

If you believe that this document breaches copyright please contact us at:

openaccess@tue.nl

providing details and we will investigate your claim. 


\title{
Equilibrated Charge Carrier Populations Govern Steady-State Nongeminate Recombination in Disordered Organic Solar Cells
}

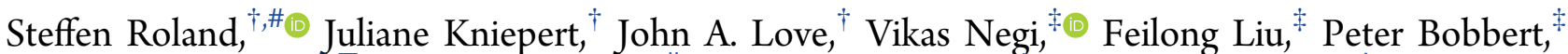

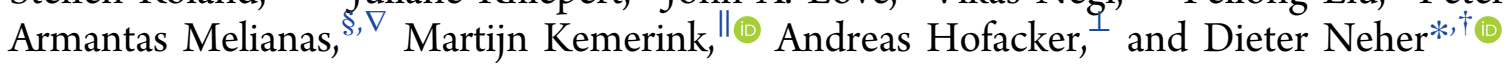 \\ ${ }^{\dagger}$ Department of Physics and Astronomy, Universität Potsdam, 14476 Potsdam, Germany \\ ${ }^{\ddagger}$ Molecular Materials and Nanosystems, Department of Applied Physics, Eindhoven University of Technology, P.O. Box 513, 5600 \\ MB Eindhoven, The Netherlands \\ ${ }^{\S}$ Biomolecular and Organic Electronics, Department of Physics, Chemistry and Biology, Linköping University, 58183 Linköping, \\ Sweden \\ "Complex Materials and Devices, Department of Physics, Chemistry and Biology, Linköping University, 58183 Linköping, Sweden \\ ${ }^{\perp}$ Dresden Integrated Center for Applied Physics and Photonic Materials (IAPP) and Institute for Applied Physics, Technische \\ Universität Dresden, 01187 Dresden, Germany
}

Supporting Information

ABSTRACT: We employed bias-assisted charge extraction techniques to investigate the transient and steady-state recombination of photogenerated charge carriers in complete devices of a disordered polymer-fullerene blend. Charge recombination is shown to be dispersive, with a significant slowdown of the recombination rate over time, consistent with the results from kinetic Monte Carlo simulations. Surprisingly, our experiments reveal little to no contributions from early time recombination of nonequilibrated charge carriers to the steady-state recombination properties. We conclude that energetic relaxation of photogenerated carriers outpaces any significant nongeminate recombination under application-relevant illumination conditions. With equilibrated charges dominating the steady-state recombination, quasi-equilibrium

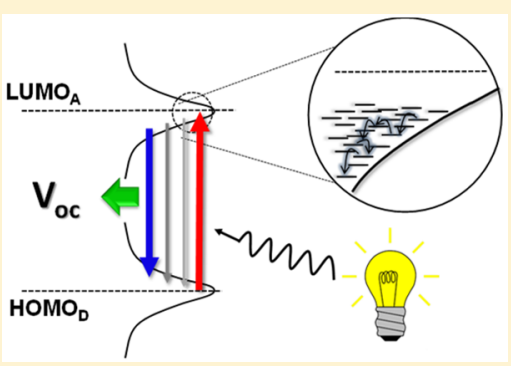
concepts appear suited for describing the open-circuit voltage of organic solar cells despite pronounced energetic disorder.

$\mathrm{T}$ here is an ongoing debate regarding the role of nonequilibrium dynamics in the extraction and recombination of photogenerated charge carriers in organic bulk heterojunction solar cells. ${ }^{1-6}$ Donor-acceptor bulk heterojunction blends possess significant positional and energetic disorder. If excitons or charges are introduced into the photoactive blend through photogeneration or photoinjection, they are very mobile initially but progressively slow down while occupying states energetically deeper and deeper in the density of states (DOS). This gives rise to so-called dispersive effects. ${ }^{7,8}$ Dispersive effects such as spectral diffusion, timedependent mobilities, and dispersive recombination have been demonstrated for a number of donor-acceptor blends. ${ }^{9-11}$ While it is generally believed that disorder is detrimental to device performance, several recent studies suggested that the dispersive charge carrier transport allows for efficient charge extraction despite low steady-state mobilities. ${ }^{1,5}$ This was rationalized by the high mobility of photogenerated charges at early time scales and their slow energetic relaxation in a broad DOS. ${ }^{8,12}$ Importantly, these results suggested that photogenerated carriers leave thin $(\sim 100 \mathrm{~nm}$ or less) devices before they have equilibrated, bringing into question the applicability of quasi-equilibrium concepts to describe the steady-state performance of disordered organic solar cells. Other studies, however, suggested that charge extraction and recombination involve mainly equilibrated excitations and charges, meaning that the physical parameters describing these processes are not explicitly time-dependent. For example, steady-state currentvoltage characteristics of organic photovoltaic devices were successfully simulated with drift-diffusion codes or modeled on the basis of quasi-equilibrium concepts. ${ }^{13,14}$ Moreover, steadystate measurements of the external quantum efficiencies of photogeneration and electroluminescence, $\mathrm{EQE}_{\mathrm{PV}}$ and $\mathrm{EQE}_{\mathrm{EL}}$ respectively, are often employed to explain the open-circuit voltage $\left(V_{\mathrm{oc}}\right)$ in donor-acceptor blends. ${ }^{15,16}$ This analysis relies on the assumption that the external bias (times the elementary charge) equals the quasi-Fermi level splitting in the bulk, which is meaningful only if most photogenerated electrons and holes are equilibrated and if these charges dominate the rate of nongeminate recombination (NGR). Given that the efficiency of state-of-the-art organic solar cells in the power-delivering regime and at open-circuit voltage conditions is limited by steady-state NRG, the understanding and control of this process is of utmost importance for the further advancement of this technology.

Received: February 22, 2019

Accepted: March 4, 2019

Published: March 4, 2019 
a)

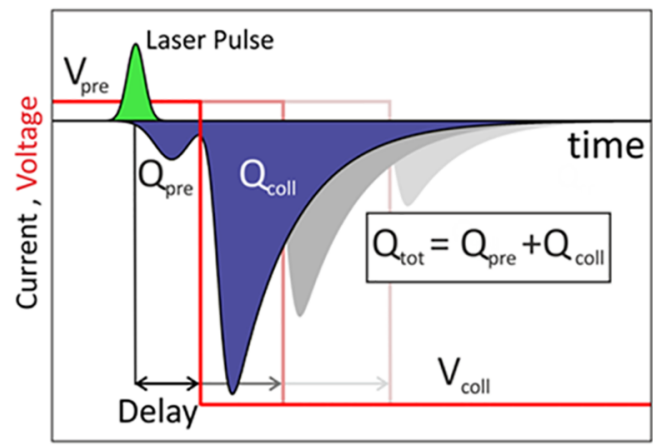

C)

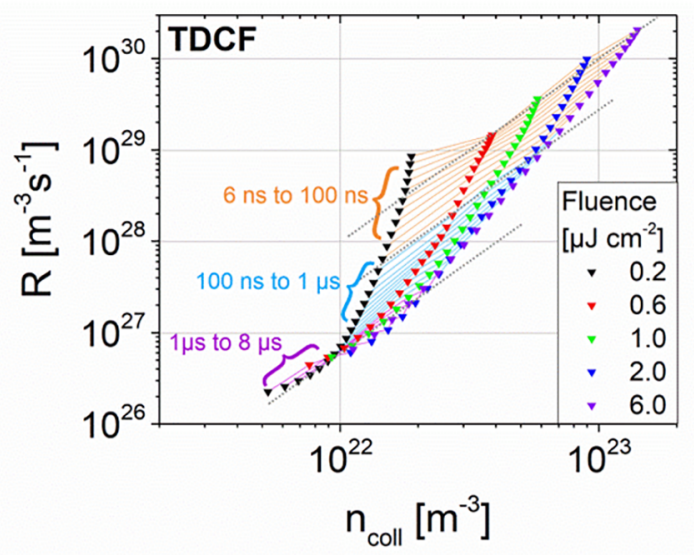

b)

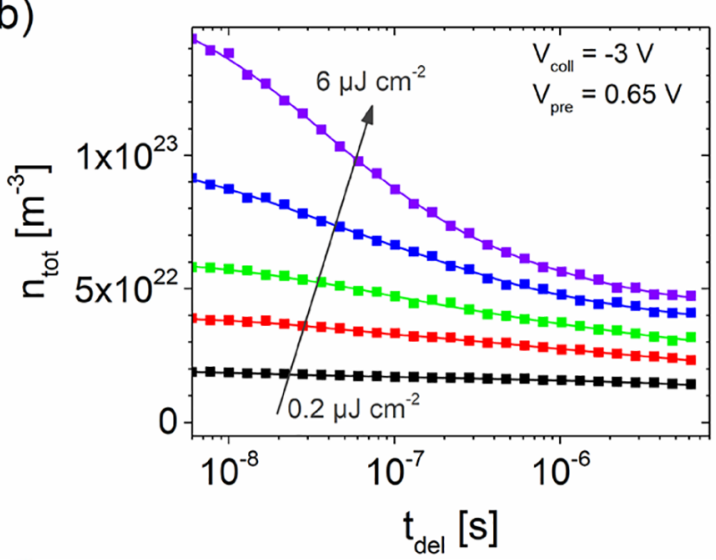

d)

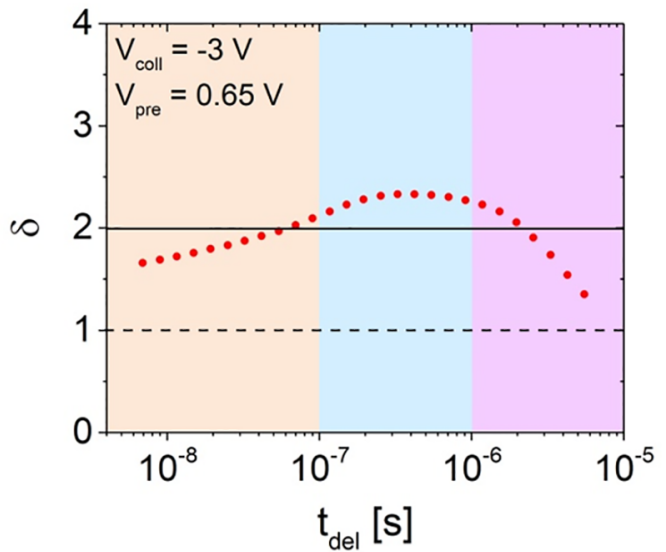

Figure 1. (a) Scheme of the transient TDCF experiment. $Q_{\text {pre }}$ and $Q_{\text {coll }}$ are the photogenerated charge extracted during the delay at a prebias $V_{\text {pre }}$ and upon application of the reverse collection bias $V_{\text {coll }}$, respectively. (b) The total charge carrier density, $n_{\text {tot }}$ (solid symbols), plotted as a function of the delay time, $t_{\mathrm{del}}$. Experiments were performed for five different fluences, with $V_{\text {pre }}=0.65 \mathrm{~V}$ and $V_{\text {coll }}=-3 \mathrm{~V}$. (c) Recombination rate versus the photogenerated carrier density, $n_{\text {coll }}$, present in the active layer at a given delay time. Thin solid lines connect recombination rates for the same delay time. Gray dotted lines indicate a recombination order of two. (d) The recombination order $\delta$ derived from panel c by power law-type fits of the equitemporal recombination rates at each delay time.

Here, we address this controversy by investigating the timedependent and steady-state NGR on the very same complete devices of a disordered polymer:fullerene blend. Our system of choice is the blend of the polymer TQ1 (poly[2,3-bis(3octyloxyphenyl)quinoxaline-5,8-diyl-alt-thiophene-2,5-diyl]) with the fullerene $\mathrm{PC}_{71} \mathrm{BM}$ ([6,6]-phenyl-C71- butyric acid methyl ester) (see Figure S1). Transient all-optical pumpprobe experiments combined with comprehensive kinetic Monte Carlo (kMC) simulations had shown that photogenerated electrons and holes are very mobile after photogeneration and that their equilibration is slow, approaching the steady-state mobilities only at the microsecond time scale. ${ }^{17}$ Explicit calculation of the photogenerated charge carrier drift distance suggests that in thin $(\sim 100 \mathrm{~nm}$ or less $)$ devices the majority $(>50 \%)$ of the photogenerated charges are extracted before their full equilibration has taken place. ${ }^{1,17}$ Transient NGR in TQ1:PCBM blends and other disordered blends has been studied before, but most of these studies relied on contact-less methods such as transient absorption spectroscopy (TAS) or time-resolved microwave conductance (TRMC) measurements. ${ }^{10,17-20}$ Other studies employed charge extraction of photogenerated carriers by linearly increasing voltage (photo-CELIV) which, however, lacks the time resolution to probe the submicrosecond time regime. ${ }^{18,21,22}$

Figure S1 shows the current density-voltage $(J-V)$ characteristics of our device, which consisted of a $150 \mathrm{~nm}$ thick TQ1:PC ${ }_{71} \mathrm{BM}$ blend sandwiched between an ITO/ PEDOT:PSS anode and a $\mathrm{Ca} / \mathrm{Al}$ or $\mathrm{LiF} / \mathrm{Al}$ cathode. Despite the rather amorphous and disordered nature of the blend, the photogenerated current approaches saturation quite rapidly, pointing to efficient extraction. This is indicative of initially very mobile charges as outlined above. Because we are interested in the comparison of transient and steady-state recombination of free charges in the very same complete device, we combined time-delayed collection field (TDCF) and bias-assisted charge extraction (BACE) experiments. Both methods employ charge extraction at reverse bias to measure the charge carrier density in the active layer. However, while TDCF uses a short laser pulse to generate a (nonequilibrated) carrier profile at a preset time and voltage, ${ }^{23,24}$ in BACE the sample is held for a sufficiently long time under constant light intensity to establish a steady-state carrier distribution. ${ }^{25}$ This allows for tracking the transient evolution of the photogenerated charge carrier density in the device and investigating the time- and density-dependent transient recombination at application-relevant carrier densities, as well as comparing transient and steady-state device behavior.

Figure 1a shows a sketch of the TDCF measurement. Charges are generated with a 5 ns laser pulse while the sample is held at a prebias, $V_{\text {pre }}$. Charges surviving NGR during the delay time, $t_{\mathrm{del}}$, are then rapidly extracted from the active layer by applying a high reverse collection voltage, $V_{\text {coll }}$. For the 
a)

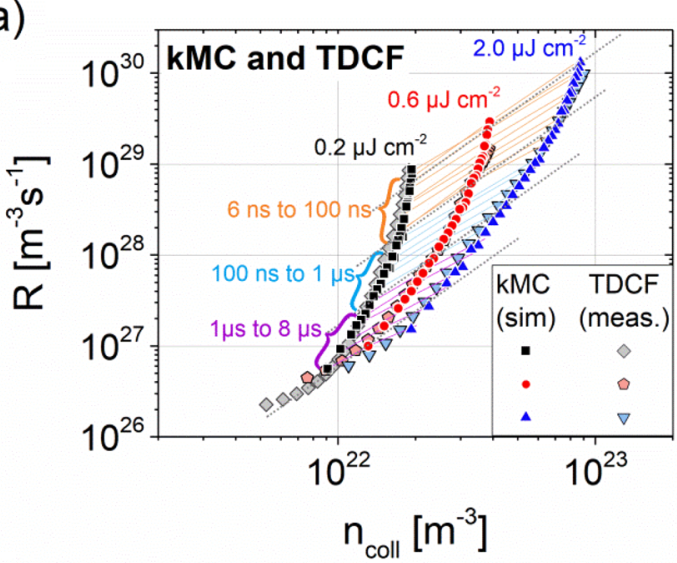

c)

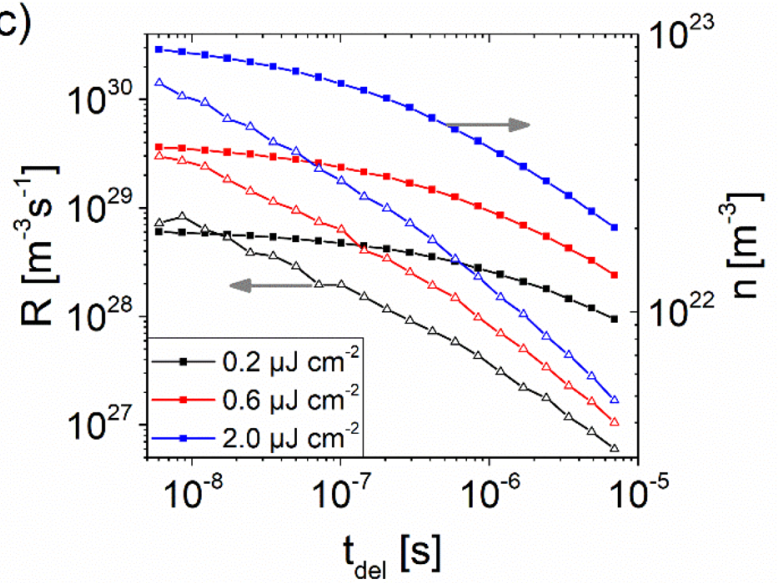

b)

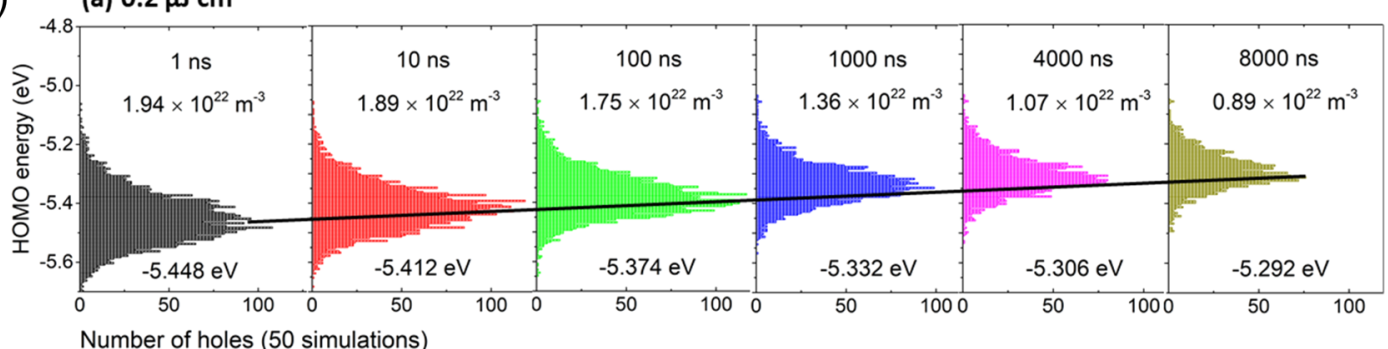

(b) $2 \mu \mathrm{cm}^{-2}$

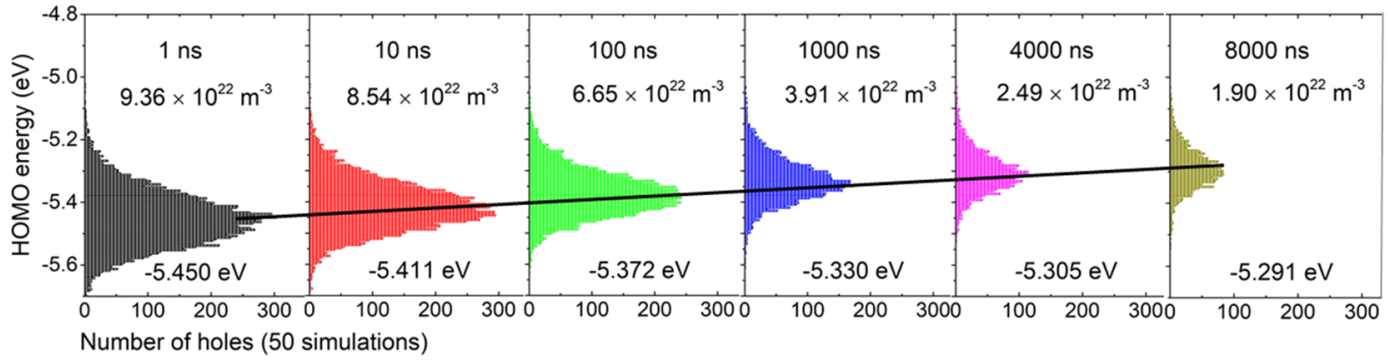

Figure 2. (a) Recombination rate as a function of carrier density from kMC simulations, compared to the measured TDCF data for the same fluences (initial charge carrier density for kMC). Thin lines connect the equitemporal data points in different time ranges (orange, 6-100 ns; blue, $100 \mathrm{~ns}$ to $1 \mu \mathrm{s}$; purple, $1-8 \mu \mathrm{s}$ ). (b) Distribution of site energies occupied by holes at different time intervals obtained from kMC simulations for a fluence of 0.2 and $2 \mu \mathrm{J} \mathrm{cm}^{-2}$. The delay time, hole density, and mean energy are shown in the graphs. The black line is a guide to the eye and indicates the energy relaxation as time progresses. (c) $R$ and $n$ plotted as functions of delay time for three characteristic fluences.

analysis of the transient recombination properties, two quantities were measured as a function of delay time: the density of photogenerated charge carriers that exit the device before the extraction voltage is applied $\left(n_{\text {pre }}\right)$ and the density of photogenerated charge carriers that are still present in the device at time $t_{\text {del }} \quad\left(n_{\text {coll }}\right)$. Therefore, $n_{\text {tot }}\left(t_{\text {del }}\right)=n_{\text {pre }}\left(t_{\text {del }}\right)+n_{\text {coll }}\left(t_{\text {del }}\right)$ is charge carrier density that survived recombination between photoexcitation and the onset of collection at $t_{\text {del }}$ (Figure 1a). Figure $1 \mathrm{~b}$ plots $n_{\text {tot }}$ as a function of delay time for different fluences. The figure reveals a continuous decrease of $n_{\text {tot }}$ with $t_{\text {del }}$ for all fluences throughout the entire time window. Using lower fluences slows recombination, meaning that recombination is indeed of higher order (see also the normalized data in Figure S2). In addition, Figure S2 indicates an initial first-order loss, which may be caused by the nongeminate recombination of highly mobile photogenerated carriers with dark-injected charge. ${ }^{12}$

Knowing that $n_{\text {tot }}$ is the photogenerated charge that survived recombination during the delay time $t_{\text {del }}$ allowed us to calculate the recombination rate $R$ via $R=\Delta n_{\text {tot }} / \Delta t_{\text {del }}$, as described before. $^{11}$ Because the dark-injected charge $\left(n_{\mathrm{dk}}=7 \pm 1 \times 10^{20} \mathrm{~m}^{-3}\right.$ at a prebias of $0.65 \mathrm{~V}$ as determined with dark-BACE) is much smaller than $n_{\text {tot }}$ for all fluences and delay times tested here, recombination with dark-injected charge is neglected in the following analysis. In Figure 1c the recombination rate $R$ is plotted as a function of the collected charge carrier density $n_{\text {coll }}$ which is an estimate of the photogenerated charge present in the sample at time $t_{\text {del }}$. The data show all characteristics of dispersive recombination in a Gaussian DOS distribution. ${ }^{26}$ First, $R$ is an explicit function of $t_{\text {del, }}$ approaching a common $R(n)$ dependence only at long delays. Second, the drop of $R$ with decreasing $n$ is most pronounced at early times (within the first $100 \mathrm{~ns}$ ) and is the steepest for the lowest fluence trace. This is due to the fact that dispersive recombination depends on carrier density, $n$, through a time-dependent recombination coefficient, $\gamma(t)$, and recombination order, $\delta(t)$ :

$$
R=\gamma(t) n^{\delta(t)}
$$



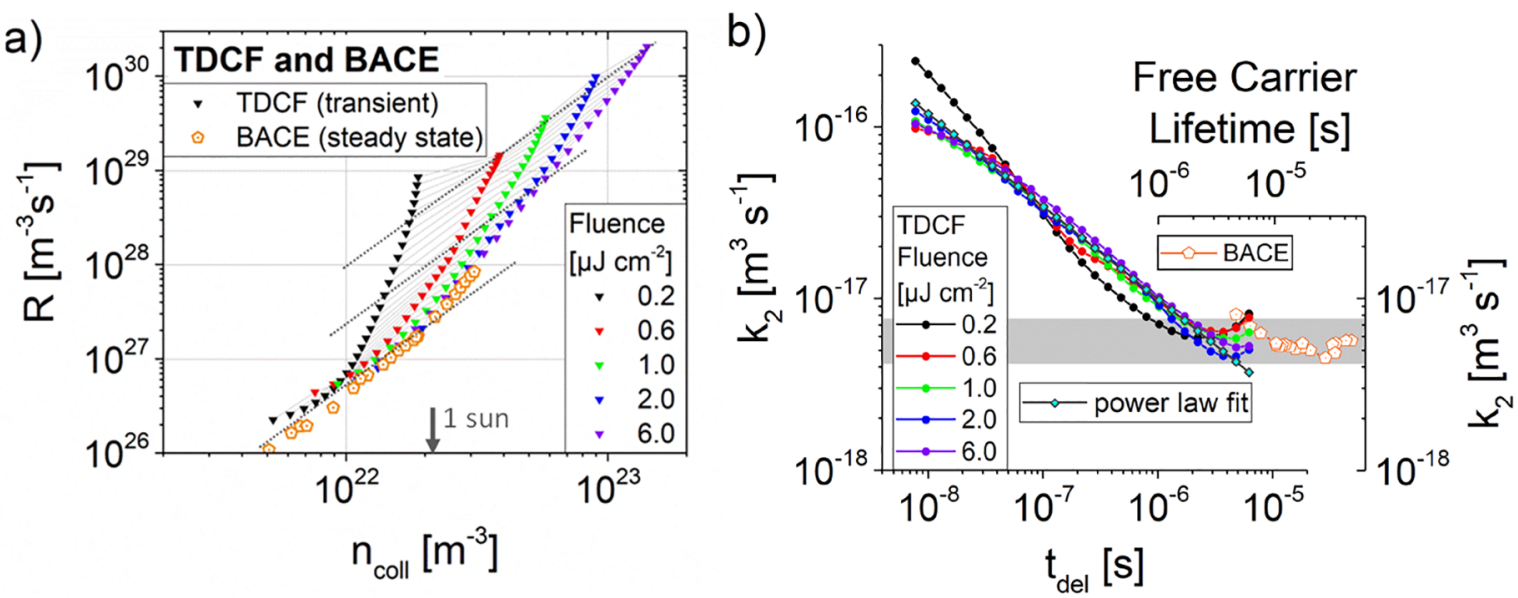

Figure 3. (a) Comparison between transient recombination rates deduced from transient TDCF experiments (solid triangles, data taken from Figure $2 \mathrm{~b}$ ) and the steady-state recombination rates taken from BACE experiments (orange circles). (b) The apparent bimolecular recombination coefficient $k_{2}$ calculated from the measured recombination rates via $R=k_{2} n^{2}$. The bimolecular recombination coefficient is plotted as a function of the delay time and the carrier lifetime for the transient (filled symbols) and steady-state (open orange symbols) data, respectively.

At low initial carrier densities, $R$ decreases rapidly by the relaxation of charge carriers, leaving insufficient time to cause an appreciable decay of the carrier density $n$. Higher carrier densities, on the other hand, will speed up recombination through the power law dependence on $n$, now competing with equilibration and causing the observed early time loss in $n_{\text {coll }}$ in the high fluence traces.

Because of this, the evaluation of $R$ versus $n$ along a given trace in terms of a time-dependent recombination order $\delta(t)$ is not meaningful. Instead, $\delta(t)$ must be retrieved from power law-type fits to equitemporal recombination data, recorded at the same delay time but with increasing fluence (as indicated by the equitemporal thin solid lines in Figure 1c). ${ }^{11,27}$ Resulting values of $\delta$ are shown in Figure $1 \mathrm{~d}$ as a function of the time after photoexcitation. Within the main range of carrier equilibration, $\delta$ varies between 1.5 and 2.3, with possible contributions from first-order recombination at the early times as noted above. A recombination order of 2 is characteristic for recombination of equilibrated charge in a Gaussian distribution of states and is also expected when considering the recombination of nonequilibrated carriers as outlined below. We noticed a gradual decay of $\delta$ for delay times beyond $1 \mu \mathrm{s}$, when most photogenerated charges have been extracted or have recombined, ${ }^{1}$ indicating a possible contribution from trap-assisted recombination of the charges deep in the DOS.

To support our interpretation of dispersive recombination in a Gaussian DOS, our experimental study has been complemented by kMC simulations based on the Gaussian disorder model. ${ }^{8,28}$ Such simulations have previously been used to describe charge carrier motion in a variety of disordered organic semiconductors. ${ }^{1,29-33}$ Details about our kMC method can be found in the Supporting Information. Simulations begin with free charge carriers randomly distributed at different sites of a simple cubic lattice, where the site energies are randomly drawn from a Gaussian distribution with a given width (standard deviation) $\sigma$. These carriers are allowed to hop to neighboring sites with a rate given by the Miller-Abrahams formalism. ${ }^{34}$ The attempt-to-hop frequencies for electrons $\left(\nu_{\mathrm{e}}\right.$ $\left.=10^{13} \mathrm{~s}^{-1}\right)$ and holes $\left(\nu_{\mathrm{h}}=10^{10} \mathrm{~s}^{-1}\right)$ are similar to those used in previous $\mathrm{kMC}$ studies of the TQ1:PCBM blend, ${ }^{35}$ but we needed to employ a greater width of the highest occupied molecular orbital (HOMO) and lowest unoccupied molecular orbital (LUMO) DOS of $150 \mathrm{meV}$ (compared to $\sigma_{\mathrm{LUMO}}=125$ $\mathrm{meV}$ and $\sigma_{\text {HOMO }}=113 \mathrm{meV}$ in ref 35$)$. When considering this discrepancy, we emphasize that our $\sigma$ 's parametrize the energetic spread of states involved in the nongeminate charge carrier recombination at the considered time scale, which is from a few nanoseconds to microseconds. The situation is clearly different from ref 35 which deals with the motion and extraction of photogenerated charges. That and other studies $^{1,10,17}$ revealed a pronounced equilibration of photogenerated electrons and holes on the subnanosecond time scale, well before NGR becomes significant, and which is therefore not captured by our kMC simulation. Within these limitations, kMC provides a very good fit to the experimental data, as can be seen from Figure 2a. Not only does kMC reproduce the rapid drop of the recombination rate by 3 orders of magnitude within the considered time window, but also it reveals the nearly second-order recombination as seen in the experiment. The corresponding time-dependent recombination order $\delta$ is deduced from linear fits of the equitemporal simulated recombination data, as for the experimental data in Figure 1, and plotted as a function of delay time in Figure S3, together with the previously shown TDCF data.

Complementary to the experiments, $\mathrm{kMC}$ is able to provide data not accessible through TDCF. As an example, Figure $2 b$ plots the density of occupied states distribution (DOOS) in the HOMO for selected delay times (see Figure S4a,b for the corresponding LUMO data). Two findings are noteworthy here. First, the energetic carrier distribution remains rather narrow and moves to higher hole energies as a total, without an appreciable broadening. This is in concert with the concept of a demarcation energy $\varepsilon^{*}$, which assumes that most carriers are situated close to a well-defined, though time-dependent, energy in the tail of the DOS. ${ }^{12,26,36}$ It is this energy that determines the speed of recombination through the rate of thermal excitation into mobile states via

$$
n_{\text {mobile }}\left(E_{\mathrm{m}}\right)=n_{\mathrm{t}} \times \frac{g\left(E_{\mathrm{m}}\right)}{g\left(E^{*}(t)\right)} \exp \left[-\frac{E_{\mathrm{m}}-\varepsilon^{*}(t)}{k_{\mathrm{B}} T}\right]
$$

where $n_{\text {mobile }}$ and $n_{\mathrm{t}}$ are the density of mobile and trapped change, respectively; $E_{\mathrm{m}}$ is the energy of mobile carriers, and $g$ is the DOS at the respective energy. Notably, for a given delay 
time, $n_{\text {mobile }}$ is strictly proportional to $n_{\mathrm{t}}$, which explains why recombination of nonequilibrated carriers depends quadratically on carrier density over eight orders in time, independent of the exact shape of the DOS. Second, it is only at larger delay times $(>\mu \mathrm{s})$ that recombination causes a significant reduction of carrier density, while equilibration occurs mostly in the early time range. This situation is summarized in Figure $S 4 c, d$, which plots the center of the DOOS (which we assign to the demarcation energy $\varepsilon^{*}$ ), and in Figure $2 c$, which shows the recombination rate and the carrier density as a function of time after photoexcitation. Notably, $\varepsilon^{*}$ decays in a very similar fashion with time for all initial carrier densities, in full accordance with conclusions from earlier experimental work reporting that the fluence has no influence on the energetic relaxation of carriers and that the initial carrier density does not affect the time dependence of the mobility. ${ }^{17}$

Having confirmed that recombination of charges photogenerated by a short laser pulse is dispersive, we now turn to the steady-state recombination properties. To do so, the carrier density in the device under steady-state illumination at opencircuit conditions was determined through BACE measurements, using a collection bias $V_{\text {coll }}$ of $-3 \mathrm{~V}$. In a BACE experiment, the solar cell is held at $V_{\text {oc }}$ under steady-state illumination by a laser diode. After the laser is switched off, the charge present in the active layer is extracted via a high reverse voltage pulse. ${ }^{13,25}$ Because recombination balances generation at $V_{o c}$ the analysis of these data reveals the steady-state recombination rate as a function of the steady-state carrier density. Details about the experimental setup and the data evaluation can be found in the Supporting Information (Figure S5). The resulting dependence of the recombination rate, $R$, on the steady-state carrier density, $n$, is plotted as open circles in Figure 3a. The data show strict second-order steady-state recombination at intermediate carrier densities, characteristic of the recombination of equilibrated charge carriers in a Gaussian DOS. ${ }^{26}$ Density-independent bimolecular recombination coefficients have been reported before and were assigned to the nongeminate recombination of relaxed carriers. $^{13,37,38}$ We also note a slight increase of the recombination order toward the highest carrier densities, which may have its origin at the occupation of higher-lying states in the DOS and a concurrent increase in the carrier mobility. More importantly, the steady-state recombination data match the transient recombination rates recorded with TDCF at long delay times, despite the 2-3 orders of magnitude higher recombination rate at early times. This is the central result of this publication. We conclude that nonequilibrated carriers do not contribute noticeably to the recombination of free charge under steady-state illumination conditions, even in the presence of significant energetic disorder.

With a second-order process governing transient and steadystate recombination at the relevant time and density scale, we can now compare these data sets in terms of the bimolecular recombination coefficient $k_{2}$ via $R=k_{2} n^{2}$. This comparison is shown in Figure $3 b$, where the time scale of steady-state recombination is defined by the carrier lifetime $\tau$ via $R=n / \tau$. The graph reveals the same power law decay $k_{2}(t)=k_{2}\left(t_{0}\right) \times\left(t / t_{0}\right)^{-\alpha}$, with $\alpha=0.54 \pm 0.4$, for all initial fluences (except at early times of the $0.2 \mu \mathrm{J} / \mathrm{cm}^{2}$ trace). It is only at the microsecond range that $k_{2}$ assumes the steady-state $k_{2}=(5.5 \pm 0.15) \times 10^{-18} \mathrm{~m}^{3} \mathrm{~s}^{-1}$. With that, the overall dynamics perfectly resemble the results from transient charge transport measurements and simulations on the same blend, which showed a pronounced relaxation of the electron and hole mobilities over several orders in magnitude and time, ${ }^{17}$ finally approaching the mobility of equilibrated (injected) charges. ${ }^{35}$ On the other hand, our early time transient data differ distinctly from the results of earlier recombination studies on TQ1:PC ${ }_{71} \mathrm{BM}$ blends. For example, contact-less transient microwave conductivity studies at the 30-600 ns scale were explained with a time-independent recombination coefficient. ${ }^{18}$ By contrast, the analysis of TAS measurements on TQ1: $\mathrm{PC}_{71} \mathrm{BM}$ films (without electrodes) indicated carrier equilibration over the entire studied time range but needed to assume that the recombination coefficient depends on the starting carrier concentration. ${ }^{19}$ Finally, results from combined TAS and photo-CELIV experiments on complete devices at low fluences were consistent with a time-independent recombination coefficient, while fitting also the high fluence data was possible only when using a power-law decay of the mobility. ${ }^{22}$ Regarding the steady-state recombination properties of the TQ1:PCBM blend, our BACE measurements reveal second-order recombination coefficients $k_{2}$ that are virtually independent of carrier lifetime and concentration (indicated in Figure $3 \mathrm{~b}$ by the shaded gray area). The $k_{2}$ from our BACE experiments compares well with the results from photo-CELIV measurements on the TQ1:PCBM blend at the $10^{-5}$ to $10^{-3} \mathrm{~s}$ time scale. ${ }^{18,22}$ The fact that the steady-state recombination rate equals the transient rate at long delays provides again support for our conclusion that steady-state NGR does not suffer from early time nonequilibrium carrier recombination processes under typical operational conditions, otherwise the steady-state value would sit above the transient long-delay limit. Earlier transient spectroscopy measurements and kMC simulations on the amorphous blend PCDTBT:PCBM showed that recombination in the absence of an electric field sets in when equilibration has largely completed. ${ }^{10}$

To conclude, while charge extraction in amorphous and disordered TQ1:PC ${ }_{71} \mathrm{BM}$ blends benefits from the rapid motion of nonequilibrated carriers, our work reveals a nearly negligible contribution of early time carrier recombination to the steady-state recombination rate. In contrast to charge extraction, NGR is a higher-order process, and fast recombination of carriers requires a high enough density of recombination partners to be present in the active layer (the recombination-equilibration balance is a function of carrier density). According to our BACE measurements, the carrier density at $V_{\mathrm{OC}}$ under one sun steady-state illumination is ca. 2 $\times 10^{22} \mathrm{~m}^{-3}$, corresponding to the initial carrier density of our lowest fluence trace where early time recombination is nearly absent (Figures $3 \mathrm{~b}$ and S5b). This leads us to conclude that the energetic relaxation of photogenerated charges in the studied TQ1:PC $\mathrm{P}_{71} \mathrm{BM}$ blend proceeds at a much higher rate than their nongeminate recombination, when considering application-relevant carrier densities. In addition, while photogenerated carriers in a transient experiment are of the same age, the age distribution of carriers under steady-state illumination is very broad, with only a small fraction of freshly generated carriers. Finally, extraction and reinjection of photogenerated carriers via one or both electrodes may provide an additional pathway for fast equilibration of the overall carrier population in the device, in particular at early times when the carriers are highly mobile. 
a)

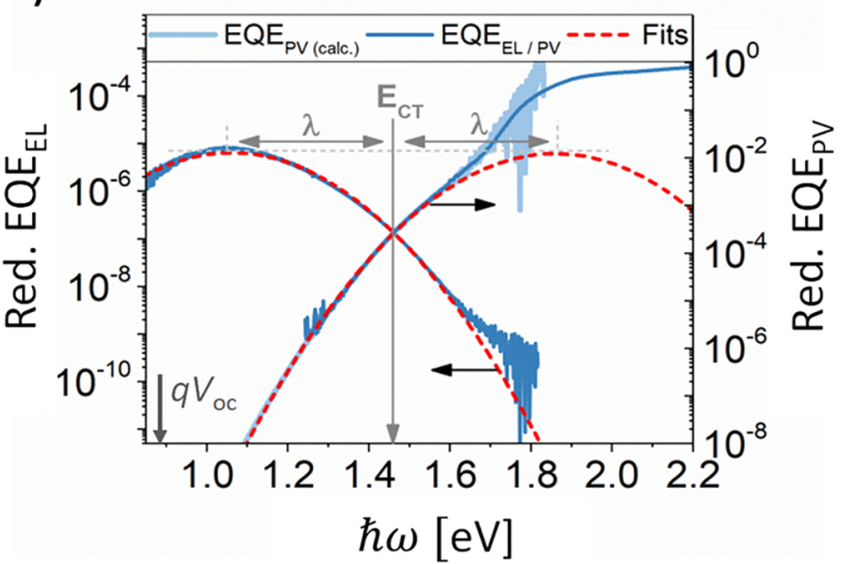

b)

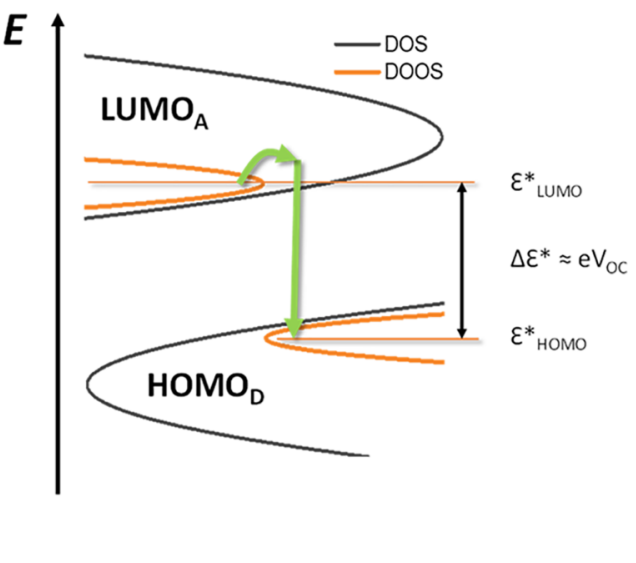

Figure 4. (a) Reduced electroluminescence and photovoltaic $\mathrm{EQE}$ spectra. The $\mathrm{EQE}_{\mathrm{PV}}$ spectrum calculated via the reciprocity relation from the depicted $\mathrm{EQE}_{\mathrm{EL}}$ spectrum is given in light blue. Red lines show Gaussian fits to the reduced $\mathrm{EQE}_{\mathrm{EL}}$ and $\mathrm{EQE}_{\mathrm{PV}}$ spectra. (b) Schematic representation of the DOS of the donor-HOMO and the acceptor-LUMO (with gray lines on a logarithmic scale) and the respective DOOS (in orange) after steady-state conditions have been nearly established. Thermalization causes a large down (up) shift of the center of the photogenerated electron (hole) population with respect to the LUMO (HOMO) energy. A possible recombination pathway comprising the thermal excitation of an electron in the acceptor LUMO into mobile states and the subsequent recombination with a hole in the donor HOMO is shown by green arrows.

If nongeminate recombination under constant illumination is mostly through equilibrated carriers, the kinetics and energetics of this process must follow quasi-equilibrium thermodynamic theories. We, therefore, tested whether the measured $V_{\mathrm{oc}}$ of this blend is in accordance with the approaches by $\mathrm{Rau}^{15}$ and by Vandewal et al., ${ }^{16}$ meaning that its value can be accurately reconstructed from the steady-state photovoltaic (PV) and electroluminescence (EL) properties. Figure $4 \mathrm{a}$ shows the reduced PV and EL quantum efficiency $\left(\mathrm{EQE}_{\mathrm{PV}}\right.$ and $\left.\mathrm{EQE}_{\mathrm{EL}}\right)$ spectra, plotted according to the analysis scheme by Vandewal et al. ${ }^{16}$ To fulfill quasi-equilibrium conditions the EL was measured under low injection conditions, close to and even slightly below $V_{\text {oc }}$ (see Figure S6). The EL spectra are independent of the driving conditions, perfectly Gaussian-shaped, and red-shifted from the emission properties of the pure components. This indicates that electroluminescence originates entirely from radiative recombination of charge transfer (CT) states. Gaussian fits to the reduced $\mathrm{EQE}_{\mathrm{EL}}$ and $\mathrm{EQE}_{\mathrm{PV}}$ spectra yield a CT-state energy $E_{\mathrm{CT}}$ of $1.46 \mathrm{eV}$ and a reorganization energy $\lambda$ of $0.4 \mathrm{eV}$. The value of the reorganization energy that is deduced from the spectral analysis already incorporates the energetic disorder of the CT state manifold as pointed out by Burke et al. ${ }^{39}$ Hence, the rather high value of the reorganization energy is in good agreement with the broad DOS distributions in this disordered system. More importantly, the $\mathrm{EQE}_{\mathrm{PV}}$ and $\mathrm{EQE}_{\mathrm{EL}}$ spectra follow Rau's reciprocity $\operatorname{EQE}_{\mathrm{EL}}(\hbar \omega) \propto$ $\mathrm{EQE}_{\mathrm{PV}}(\hbar \omega) \times \phi_{\mathrm{BB}}(\hbar \omega, T)$, where $\phi_{\mathrm{BB}}(\hbar \omega, T)$ is the photon flux of the blackbody spectrum at temperature $T .^{15,16}$ This is shown with the light blue line in Figure 4a, where $T=300 \mathrm{~K}$, only slightly above room temperature. Rau's reciprocity was derived under the condition of thermal equilibrium, where all carriers and excitations are quasi-equilibrated. The fulfillment of this reciprocity for our TQ1:PCBM blend, therefore, suggests that EL originates mostly from an (injected) equilibrated population of free carriers, possibly involving thermal excitations to higher states. ${ }^{40}$

Accordingly, if the steady-state recombination of photogenerated carriers in the illuminated solar cell is likewise dominated by equilibrated populations, the measured $V_{\mathrm{oc}}$ must agree with the value predicted by Rau:

$$
V_{\mathrm{OC}}=V_{\mathrm{OC}, \mathrm{rad}}+\frac{k_{\mathrm{B}} T}{q} \ln \left(\mathrm{EQE}_{\mathrm{EL}}\right)
$$

Here, $\mathrm{EQE}_{\mathrm{EL}}$ is the integrated external quantum efficiency of $\mathrm{EL}$, which is $2.6 \times 10^{-6}$ at low injection conditions, corresponding to a nonradiative $V_{\mathrm{oc}}$ loss of $0.325 \mathrm{~V}$ (see Figure S6). $V_{\mathrm{OC} \text {,rad }}$ is the radiative $V_{\mathrm{oc}}$-limit, which is calculated from

$$
V_{\mathrm{OC}, \mathrm{rad}}=\frac{k_{\mathrm{B}} T}{q} \ln \left(\frac{J_{\mathrm{G}}}{J_{0, \mathrm{rad}}}+1\right)
$$

where $J_{\mathrm{G}}$ is the photogenerated current density (which we set equal to $J_{\mathrm{SC}}$ ) and $J_{0, \text { rad }}$ is the radiative dark current given by $J_{0, \text { rad }}=q \int \mathrm{EQE}_{\mathrm{PV}}(E) \Phi_{\mathrm{BB}}(E) \mathrm{d} E$. Using the steady-state $\mathrm{EQE}_{\mathrm{PV}}$ spectra, we arrive at $V_{\mathrm{OC}, \mathrm{rad}}=1.22 \mathrm{~V}$, which combined with a nonradiative $V_{\mathrm{oc}}$ loss of $0.325 \mathrm{~V}$ yields a predicted $V_{\mathrm{oc}}$ of $0.895 \mathrm{~V}$. This value is in excellent agreement with the measured $V_{\text {oc }}$ of $0.893 \mathrm{~V}$ under simulated AM1.5G illumination. This very good agreement provides further evidence that at $V_{\text {oc }}$ recombination of photogenerated charges is indeed taking place via quasi-equilibrated carrier populations, despite a large energetic disorder in this highly disordered polymer:fullerene blend.

With a $V_{\text {oc }}$ of around $0.89 \mathrm{~V}, \mathrm{q} V_{\text {oc }}$ lies far below the optical gap and the CT energy, suggesting a significant $V_{\text {oc }}$ loss in our system as a consequence of energetic disorder and charge carrier equilibration. ${ }^{26,39,41-43}$ This large loss is also evident from the $\mathrm{kMC}$ simulations. At 1 sun equivalent illumination, the steady-state carrier density and recombination rate are $n_{1 \text { sun }} \approx 2.1 \times 10^{22} \mathrm{~m}^{-3}$ and $R_{1 \text { sun }} \approx 1.8 \times 10^{27} \mathrm{~m}^{-3} \mathrm{~s}^{-1}$, respectively. Considering the transient recombination trace for a fluence of $2 \mu \mathrm{J} \mathrm{cm}^{-2}$, such conditions are attained after ca. $2 \mu \mathrm{s}$. At this point, the difference between the demarcation energy in the LUMO and HOMO, $\Delta \varepsilon^{*}=\epsilon_{\mathrm{LUMO}}^{*}-\epsilon_{\mathrm{HOMO}}^{*}$ has 
decreased to approximately $0.7 \mathrm{eV}$, which is $0.9 \mathrm{eV}$ smaller than the LUMO-HOMO gap assumed in the simulation. When comparing the measured $V_{\text {oc }}$ to $\Delta \varepsilon^{*}$ from the simulation, it is important to remember that the latter depends on our choice of the LUMO and HOMO energy of $-4.1 \mathrm{eV}$ and $-5.7 \mathrm{eV}$, respectively, which was taken from previous work. ${ }^{17}$ Various groups reported a PCBM LUMO energy of $-3.9 \mathrm{eV}$ and above, ${ }^{4-46}$ depending (among other factors) on the degree of fullerene aggregation. Reasonable agreement between $V_{\text {oc }}$ and $\Delta \varepsilon^{*}$ would be found for a PCBM LUMO energy of $-3.9 \mathrm{eV}$. This situation is schematically depicted in Figure $4 \mathrm{~b}$.

In conclusion, we studied transient and steady-state charge recombination on complete devices of the amorphous and disordered TQ1:PC ${ }_{71} \mathrm{BM}$ blend, using time-delayed collection field and bias-assisted charge extraction measurements in combination with kinetic Monte Carlo simulations. The results reveal a pronounced slowdown of nongeminate recombination on the nanosecond to microsecond time scale, which we assign to the energetic relaxation of photogenerated charges. On the other hand, nonequilibrated charge carriers are shown to contribute negligibly to nongeminate recombination under continuous illumination. Consequently, while nonequilibrium charge carrier dynamics governs charge extraction in such amorphous solar cells as reported in previous publications, our work shows that steady-state recombination properties are determined by equilibrated charge carrier populations. Energetic disorder is, therefore, beneficial when aiming at a favorable extraction-recombination balance, as proposed recently, but this may come at the expense of significant $V_{\text {oc }}$ losses due to the equilibration of charges and excitations in the inhomogeneously broadened DOS. Finally, our finding that steady-state recombination involves mostly equilibrated charges rationalizes the use of quasi-equilibrium concepts to describe the open-circuit voltage of organic solar cells despite pronounced energetic disorder and dispersive effects.

\section{ASSOCIATED CONTENT}

\section{S Supporting Information}

The Supporting Information is available free of charge on the ACS Publications website at DOI: 10.1021/acs.jpclett.9b00516.

Description of the sample preparation and $J-V$ characteristics; technical details about the TDCF measurements and exemplary data sets; further details about the kMC simulation and the simulation results and outcome; technical details about the BACE measurements and exemplary data sets; additional information on the determination of the $V_{\text {oc }}$ from the $\mathrm{EQE}_{\mathrm{PV}}$ and $\mathrm{EQE}_{\mathrm{EL}}$ measurements (PDF)

\section{AUTHOR INFORMATION}

\section{Corresponding Author}

*E-mail: neher@uni-potsdam.de.

\section{ORCID}

Steffen Roland: 0000-0002-8299-5560

Vikas Negi: 0000-0003-1240-3109

Martijn Kemerink: 0000-0002-7104-7127

Dieter Neher: 0000-0001-6618-8403

\section{Present Addresses}

${ }^{\#}$ S.R.: UP Transfer GmbH, Am Neuen Palais 10, 14469 Potsdam.

$\nabla$ A.M.: Department of Materials Science and Engineering, Stanford University, Stanford, CA 94305.

\section{Notes}

The authors declare no competing financial interest.

\section{ACKNOWLEDGMENTS}

The authors thank Prof. Dr. Koen Vandewal (TU Dresden, now Hasselt University) and Prof. Dr. Thomas Kirchartz (FZ Jülich) for fruitful discussions. We also acknowledge Jona Kurpiers and Dr. Frank Jaiser (U Potsdam) for helping with the TDCF and the BACE setup and the data analysis. This work was funded by the German Ministry of Science and Education (BMBF) within the project UNVEIL and by the German Science Foundation (INST 336/94-1 FUGG and NE410/15-1). The work is part of the Industrial Partnership Programme (IPP) "Computational sciences for energy research" of The Netherlands Organisation for Scientific Research Institutes (NWO-I). This research programme is cofinanced by Shell Global Solutions International B.V. A.M. gratefully acknowledges support from the Knut and Alice Wallenberg Foundation (KAW 2016.0494) for Postdoctoral Research at Stanford University.

\section{REFERENCES}

(1) Melianas, A.; Pranculis, V.; Devizis, A.; Gulbinas, V.; Inganäs, O.; Kemerink, M. Dispersion-Dominated Photocurrent in Polymer:Fullerene Solar Cells. Adv. Funct. Mater. 2014, 24, 4507-4514.

(2) Philippa, B.; Stolterfoht, M.; Burn, P. L.; Juška, G.; Meredith, P.; White, R. D.; Pivrikas, A. The Impact of Hot Charge Carrier Mobility on Photocurrent Losses in Polymer-Based Solar Cells. Sci. Rep. 2015, 4, 5695.

(3) van der Kaap, N. J.; Koster, L. J. A. Charge Carrier Thermalization in Organic Diodes. Sci. Rep. 2016, 6, 19794.

(4) Brigeman, A. N.; Fusella, M. A.; Rand, B. P.; Giebink, N. C. Nonthermal Site Occupation at the Donor-Acceptor Interface of Organic Solar Cells. Phys. Rev. Appl. 2018, 10, 034034.

(5) Gasparini, N.; Salvador, M.; Heumueller, T.; Richter, M.; Classen, A.; Shrestha, S.; Matt, G. J.; Holliday, S.; Strohm, S.; Egelhaaf, H. J.; et al. Polymer:Nonfullerene Bulk Heterojunction Solar Cells with Exceptionally Low Recombination Rates. Adv. Energy Mater. 2017, 7, 1701561.

(6) Felekidis, N.; Melianas, A.; Aguirre, L. E.; Kemerink, M. Comment on "Charge Carrier Extraction in Organic Solar Cells Governed by Steady-State Mobilities. Adv. Energy Mater. 2018, 8, 1800419.

(7) Scher, H.; Montroll, E. W. Anomalous Transit-Time Dispersion in Amorphous Solids. Phys. Rev. B 1975, 12, 2455-2477.

(8) Bässler, H. Charge Transport in Disordered Organic Photoconductors a Monte Carlo Simulation Study. Phys. Status Solidi B 1993, 175, 15-56.

(9) Vithanage, D. A.; Devižis, a.; Abramavičius, V.; Infahsaeng, Y.; Abramavičius, D.; MacKenzie, R. C. I.; Keivanidis, P. E.; Yartsev, a.; Hertel, D.; Nelson, J.; et al. Visualizing Charge Separation in Bulk Heterojunction Organic Solar Cells. Nat. Commun. 2013, 4, 2334.

(10) Howard, I. A.; Etzold, F.; Laquai, F.; Kemerink, M. Nonequilibrium Charge Dynamics in Organic Solar Cells. Adv. Energy Mater. 2014, 4, 1301743.

(11) Kurpiers, J.; Neher, D. Dispersive Non-Geminate Recombination in an Amorphous Polymer:Fullerene Blend. Sci. Rep. 2016, 6, 26832.

(12) Orenstein, J.; Kastner, M. Photocurrent Transient Spectroscopy: Measurement of the Density of Localized States in a-As2Se3. Phys. Rev. Lett. 1981, 46, 1421-1424. 
(13) Kniepert, J.; Lange, I.; van der Kaap, N. J.; Koster, L. J. A.; Neher, D. A Conclusive View on Charge Generation, Recombination, and Extraction in As-Prepared and Annealed P3HT:PCBM Blends: Combined Experimental and Simulation Work. Adv. Energy Mater. 2014, 4, 1301401.

(14) Armin, A.; Chen, Z.; Jin, Y.; Zhang, K.; Huang, F.; Shoaee, S. A Shockley-Type Polymer: Fullerene Solar Cell. Adv. Energy Mater. 2018, 8, 1701450.

(15) Rau, U. Reciprocity Relation between Photovoltaic Quantum Efficiency and Electroluminescent Emission of Solar Cells. Phys. Rev. B: Condens. Matter Mater. Phys. 2007, 76, 085303.

(16) Vandewal, K.; Tvingstedt, K.; Gadisa, A.; Inganäs, O.; Manca, J. V. Relating the Open-Circuit Voltage to Interface Molecular Properties of Donor:Acceptor Bulk Heterojunction Solar Cells. Phys. Rev. B: Condens. Matter Mater. Phys. 2010, 81, 125204.

(17) Melianas, A.; Etzold, F.; Savenije, T. J.; Laquai, F.; Inganäs, O.; Kemerink, M. Photo-Generated Carriers Lose Energy during Extraction from Polymer-Fullerene Solar Cells. Nat. Commun. 2015, 6, 8778 .

(18) Murthy, D. H. K.; Melianas, A.; Tang, Z.; Juška, G.; Arlauskas, K.; Zhang, F.; Siebbeles, L. D. A.; Inganäs, O.; Savenije, T. J. Origin of Reduced Bimolecular Recombination in Blends of Conjugated Polymers and Fullerenes. Adv. Funct. Mater. 2013, 23, 4262-4268.

(19) Vithanage, D. A.; Wang, E.; Wang, Z.; Ma, F.; Inganäs, O.; Andersson, M. R.; Yartsev, A.; Sundström, V.; Pascher, T. Charge Carrier Dynamics of Polymer:Fullerene Blends: From Geminate to Non-Geminate Recombination. Adv. Energy Mater. 2014, 4, 1301706.

(20) Eng, M. P.; Barnes, P. R. F.; Durrant, J. R. ConcentrationDependent Hole Mobility and Recombination Coefficient in Bulk Heterojunctions Determined from Transient Absorption Spectroscopy. J. Phys. Chem. Lett. 2010, 1, 3096-3100.

(21) Deibel, C.; Baumann, A.; Wagenpfahl, A.; Dyakonov, V. Polaron Recombination in Pristine and Annealed Bulk Heterojunction Solar Cells. Synth. Met. 2009, 159, 2345-2347.

(22) Andersson, L. M.; Melianas, A.; Infahasaeng, Y.; Tang, Z.; Yartsev, A.; Inganäs, O.; Sundström, V. Unified Study of Recombination in Polymer:Fullerene Solar Cells Using Transient Absorption and Charge-Extraction Measurements. J. Phys. Chem. Lett. 2013, 4, 2069-2072.

(23) Kniepert, J.; Schubert, M.; Blakesley, J. C.; Neher, D. Photogeneration and Recombination in P3HTPCBM Solar Cells Probed by Time-Delayed Collection Field Experiments. J. Phys. Chem. Lett. 2011, 2, 700-705.

(24) Mingebach, M.; Walter, S.; Dyakonov, V.; Deibel, C. Direct and Charge Transfer State Mediated Photogeneration in PolymerFullerene Bulk Heterojunction Solar Cells. Appl. Phys. Lett. 2012, 100, 193302.

(25) Lange, I.; Kniepert, J.; Pingel, P.; Dumsch, I.; Allard, S.; Janietz, S.; Scherf, U.; Neher, D. Correlation between the Open Circuit Voltage and the Energetics of Organic Bulk Heterojunction Solar Cells. J. Phys. Chem. Lett. 2013, 4, 3865.

(26) Hofacker, A.; Neher, D. Dispersive and Steady-State Recombination in Organic Disordered Semiconductors. Phys. Rev. B: Condens. Matter Mater. Phys. 2017, 96, 245204.

(27) Paulke, A.; Stranks, S. D.; Kniepert, J.; Kurpiers, J.; Wolff, C. M.; Schön, N.; Snaith, H. J.; Brenner, T. J. K.; Neher, D. Charge Carrier Recombination Dynamics in Perovskite and Polymer Solar Cells. Appl. Phys. Lett. 2016, 108, 113505.

(28) Baranovskii, S. D. Theoretical Description of Charge Transport in Disordered Organic Semiconductors. Phys. Status Solidi B 2014, 251, 487-525.

(29) Mesta, M.; van Eersel, H.; Coehoorn, R.; Bobbert, P. A. Kinetic Monte Carlo Modeling of the Efficiency Roll-off in a Multilayer White Organic Light-Emitting Device. Appl. Phys. Lett. 2016, 108, 133301.

(30) Mesta, M.; Carvelli, M.; de Vries, R. J.; van Eersel, H.; van der Holst, J. J. M.; Schober, M.; Furno, M.; Lüssem, B.; Leo, K.; Loebl, P.; et al. Molecular-Scale Simulation of Electroluminescence in a Multilayer White Organic Light-Emitting Diode. Nat. Mater. 2013, 12, 652-658.
(31) van Eersel, H.; Bobbert, P. A.; Coehoorn, R. Kinetic Monte Carlo Study of Triplet-Triplet Annihilation in Organic Phosphorescent Emitters. J. Appl. Phys. 2015, 117, 115502.

(32) van Eersel, H.; Bobbert, P. A.; Janssen, R. A. J.; Coehoorn, R. Monte Carlo Study of Efficiency Roll-off of Phosphorescent Organic Light-Emitting Diodes: Evidence for Dominant Role of TripletPolaron Quenching. Appl. Phys. Lett. 2014, 105, 143303.

(33) Coehoorn, R.; van Eersel, H.; Bobbert, P.; Janssen, R. Kinetic Monte Carlo Study of the Sensitivity of OLED Efficiency and Lifetime to Materials Parameters. Adv. Funct. Mater. 2015, 25, 20242037.

(34) Miller, A.; Abrahams, E. Impurity Conduction at Low Concentrations. Phys. Rev. 1960, 120, 745-755.

(35) Melianas, A.; Pranculis, V.; Xia, Y.; Felekidis, N.; Inganäs, O.; Gulbinas, V.; Kemerink, M. Photogenerated Carrier Mobility Significantly Exceeds Injected Carrier Mobility in Organic Solar Cells. Adv. Energy Mater. 2017, 7, 1602143.

(36) Hofacker, A.; Oelerich, J. O.; Nenashev, A. V.; Gebhard, F.; Baranovskii, S. D. Theory to Carrier Recombination in Organic Disordered Semiconductors. J. Appl. Phys. 2014, 115, 223713.

(37) Garcia-Belmonte, G.; Boix, P. P.; Bisquert, J.; Sessolo, M.; Bolink, H. J. Simultaneous Determination of Carrier Lifetime and Electron Density-of-States in P3HT:PCBM Organic Solar Cells under Illumination by Impedance Spectroscopy. Sol. Energy Mater. Sol. Cells 2010, 94, 366-375.

(38) Guerrero, A.; Marchesi, L. F.; Boix, P. P.; Bisquert, J.; GarciaBelmonte, G. Recombination in Organic Bulk Heterojunction Solar Cells: Small Dependence of Interfacial Charge Transfer Kinetics on Fullerene Affinity. J. Phys. Chem. Lett. 2012, 3, 1386-1392.

(39) Burke, T. M.; Sweetnam, S.; Vandewal, K.; McGehee, M. D. Beyond Langevin Recombination: How Equilibrium Between Free Carriers and Charge Transfer States Determines the Open-Circuit Voltage of Organic Solar Cells. Adv. Energy Mater. 2015, 5, 1500123.

(40) Gong, W.; Faist, M. A.; Ekins-Daukes, N. J.; Xu, Z.; Bradley, D. D. C.; Nelson, J.; Kirchartz, T. Influence of Energetic Disorder on Electroluminescence Emission in Polymer: Fullerene Solar Cells. Phys. Rev. B: Condens. Matter Mater. Phys. 2012, 86, 024201.

(41) Garcia-Belmonte, G.; Boix, P. P.; Bisquert, J.; Lenes, M.; Bolink, H. J.; La Rosa, A.; Filippone, S.; Martin, N. Influence of the Intermediate Density-of-States Occupancy on Open-Circuit Voltage of Bulk Heterojunction Solar Cells with Different Fullerene Acceptors. J. Phys. Chem. Lett. 2010, 1, 2566-2571.

(42) Blakesley, J. C.; Neher, D. Relationship between Energetic Disorder and Open-Circuit Voltage in Bulk Heterojunction Organic Solar Cells. Phys. Rev. B: Condens. Matter Mater. Phys. 2011, 84, 075210.

(43) Collins, S. D.; Proctor, C. M.; Ran, N. A.; Nguyen, T.-Q. Understanding Open-Circuit Voltage Loss through the Density of States in Organic Bulk Heterojunction Solar Cells. Adv. Energy Mater. 2016, 6, 1501721.

(44) Guan, Z.-L.; Kim, J. B.; Wang, H.; Jaye, C.; Fischer, D. A.; Loo, Y.-L.; Kahn, A. Direct Determination of the Electronic Structure of the Poly(3-Hexylthiophene):Phenyl-[6,6]-C61 Butyric Acid Methyl Ester Blend. Org. Electron. 2010, 11, 1779-1785.

(45) Albrecht, S.; Vandewal, K.; Tumbleston, J. R.; Fischer, F. S. U.; Douglas, J. D.; Frechet, J. M. J.; Ludwigs, S.; Ade, H.; Salleo, A.; Neher, D. On the Efficiency of Charge Transfer State Splitting in Polymer: Fullerene Solar Cells. Adv. Mater. 2014, 26, 2533-2539.

(46) Zhong, Y.; Izawa, S.; Hashimoto, K.; Tajima, K.; Koganezawa, T.; Yoshida, H. Crystallization-Induced Energy Level Change of [6,6]-Phenyl-C 61 -Butyric Acid Methyl Ester (PCBM) Film: Impact of Electronic Polarization Energy. J. Phys. Chem. C 2015, 119, 23-28. 